\title{
CHILE: El AÑo EN QUE SAlimos A LA CALlE*
}

Chile: The year we took the streets

\section{CAROLINA SEGOVIA}

Centro de Estudios Públicos y Universidad Diego Portales

\section{RICARDO GAMBOA}

Instituto de Estudios Internacionales, Universidad de Chile

\section{RESUMEN}

En este artículo se describen y analizan los principales acontecimientos sociales y políticos que marcaron el segundo año de gobierno de Sebastián Piñera. Como veremos, el 2011 estuvo marcado por un aumento significativo de procesos de movilización social que llevaron a la calle la expresión de sus demandas sociales, y que mostraron la incapacidad del gobierno y los partidos políticos en su conjunto de hacer frente a estos nuevos desafíos. Estos acontecimientos y sus consecuencias políticas son indicios de que el sistema político chileno puede estar cambiando, generando un alto grado de incertidumbre respecto a cómo ellos se desarrollarán en el futuro.

Palabras clave: Chile, Piñera, gobierno, movimiento estudiantil.

\begin{abstract}
This article describes and analyzes the main social and political events of the second year of Sebastian Piñera's government. The year 2011 showed a significant increase of social mobilization processes, that took to the streets the expression of social demands, showing the government' and political parties' inability to cope with these new challenges. These events and their political consequences are evidence of possible changes in the Chilean political system, producing a high level of uncertainty as to how they will develop in the future.
\end{abstract}

Key words: Chile, Piñera, government, student's movement. 


\section{INTRODUCCIÓN}

El 2011 correspondió al segundo año de gobierno de Sebastián Piñera, que lidera el primer gobierno de derecha elegido democráticamente en más de 50 años, y que es apoyado por su partido, Renovación Nacional (RN) y la Unión Demócrata Independiente (UDI). Piñera triunfó en las elecciones presidenciales de 2009/2010, al obtener el 51,6\% de los votos en la segunda vuelta realizada el 17 de enero de 2010, desplazando así a la coalición que había gobernado por 20 años, la Concertación de Partidos por la Democracia. Su discurso de campaña estuvo fuertemente marcado por las promesas de introducir un alto grado de eficiencia en la gestión del aparato público, la renovación del personal político con la entrada de nuevos partidos y "nuevas caras" a la administración del Estado (que serían "los mejores"), enfrentar con decisión los principales problemas de política pública, particularmente el de la delincuencia, y que el país retomaría altos niveles de crecimiento económico (Castiglioni, 2010). Así, el inicio del gobierno tuvo lugar en un marco de altos niveles de expectativas por la prometida "nueva forma de gobernar" que promovería la excelencia en todos los niveles de la gestión pública. Estas expectativas fueron amplificadas por el propio Presidente al inicio de su gobierno, como se evidencia en su afirmación "en 20 días yo siento que hemos avanzado más que otros, tal vez en 20 años" (1 de abril de 2010).

El 2010 estuvo marcado, entonces, por el debut de este nuevo gobierno el cual debió enfrentar desde un comienzo una tarea para la cual no estaba preparado. Los efectos del terremoto y maremoto del 27 de febrero de 2010 obligaron a cambiar la agenda de gobierno, a una centrada fundamentalmente en la reconstrucción del país. Este fue el principal hecho social y político de ese año. Junto a eso, un accidente minero, producido en agosto de 2010 y que dejó atrapadas a 33 personas por un período de dos meses, concentró la discusión y el debate público durante la segunda mitad del año. El éxito en el rescate dio al gobierno un nuevo -aunque corto-impulso.

El 2011 fue distinto, ya que enfrentó a Piñera y su gobierno con importantes desafíos, no previstos. Por una parte, distintos grupos sociales hicieron uso extensivo de las manifestaciones y marchas políticas, utilizando la calle como espacio privilegiado de expresión de sus demandas, y en la que amplios grupos expresaron su crítica a la clase política, desestimándola como representativa de sus intereses. Por la otra, la "nueva forma de gobernar" y los problemas en el proceso de reconstrucción obligaron a generar algunos cambios en la forma en que se venía conduciendo el gobierno, y a una serie de cambios ministeriales que le entregaron un mayor peso político, y una -relativamejor relación con los partidos de gobierno. Desde el punto de vista de los partidos políticos, las relaciones al interior de la coalición gobernante han mostrado problemas constantes de coordinación, pero, más importante todavía, de desacuerdo frente a las tareas que el gobierno debe priorizar. La relación entre oficialismo y oposición ha sido difícil, criticando el primero constantemente el supuesto obstruccionismo legislativo de la Concertación. Finalmente, el gobierno ha visto en sus cifras de adhesión y aprobación una baja sustantiva que expresa la frustración de las expectativas generadas con la elección y el descontento con la gestión gubernamental. 


\section{Temas salientes en la realidad nacional y su impacto político}

\section{a) Coyuntura social y política}

El 2011 estuvo marcado principalmente por un aumento sustantivo del uso de la movilización social como instrumento de expresión de demandas sociales. De acuerdo a las cifras oficiales, sólo en la Región Metropolitana se autorizaron 240 marchas -versus 134 en 2010-, lo que corresponde a un promedio de 20 marchas mensuales (Pérez, 2012). Las cifras a nivel nacional, por su parte, estiman en alrededor de 6.000 manifestaciones públicas y 2.000.000 de participantes durante el año (Hinzpeter, 2011). Estos son los niveles de movilización social más altos vistos desde $1990 .{ }^{1}$ Ahora bien, más allá de la cantidad y masividad de las manifestaciones públicas, estos procesos sociales definieron la agenda política del año, razón por la cual las coyunturas social y política serán consideradas en conjunto. A continuación analizamos los aspectos más importantes en esta materia. ${ }^{2}$

En enero de 2011 se gestó el primer episodio de amplia movilización, el cual tuvo carácter local. Este se realizó en la región más austral de Chile, Magallanes, a partir del anuncio de la empresa petrolera estatal, ENAP, de que habría un alza en el precio del combustible para la región, de hasta un 16,8\%, producto principalmente de la decisión de terminar con los subsidios al consumo que beneficiaban a los habitantes de la región. ${ }^{3}$ Esto generó un fuerte conflicto entre el gobierno y la empresa, por una parte, y los ciudadanos y representantes políticos y sociales de la zona, por otra. ${ }^{4} \mathrm{El}$ argumento, desde el gobierno, fue que el alza del precio era inevitable debido a un problema de escasez del recurso, y que este aumento evitaría enfrentar problemas de abastecimiento a futuro. ${ }^{5}$ Para los habitantes de la zona, sin embargo, quienes requieren del gas para calefacción durante prácticamente todo el año, esta alza era insostenible, argumentándose la necesidad de generar políticas específicas para zonas extremas. El conflicto, que se inició con la toma del aeropuerto de Punta Arenas el 5 de enero, incluyó también amplias movilizaciones sociales en la zona, el cierre de los accesos a Punta Arenas y de pasos fronterizos. El gobierno se mostró desde un principio muy dubitativo respecto a qué hacer para destrabar el problema, lo cual sólo se logró luego del establecimiento de una mesa de diálogo que

1 Un antecedente que puede ser relevante al respecto es lo ocurrido el 2010 con ocasión de la aprobación, por las instancias correspondientes, de la construcción de una central eléctrica en el sector conocido como Punta de Choros (IV Región). Esa decisión desencadenó un enérgico rechazo de grupos medioambientalistas y de la población en general, destacando la participación de conocidos animadores de televisión. Ante esta reacción masiva, Piñera, quien en su campaña había señalado que estaba en contra del proyecto en ese lugar, "convenció" a la empresa Suez Energy de abandonar el proyecto (http:/ / www.lanacion.cl/central-termoelectrica-barrancones-no-se-construira-en-punta-de-choros / noticias/2010-08-26/113013.html).

2 Aparte de las aquí tratadas, existieron otros episodios de movilización pública, que no se analizan por razones de espacio y por considerarse menos significativas políticamente.

3 http://www.biobiochile.cl/2011/01/05/rainieri-respalda-alza-de-gas-y-cataloga-subsidio-en-magallanescomo-una-fiesta-que-debe-terminar.shtml

4 http://www.emol.com/noticias/nacional/2011/01/05/456620/punta-arenas-con-bloqueo-de-aeropuertoparte-protesta-por-alza-de-gas.html

5 http://www.emol.com/noticias/economia/2011/01/05/456644/ministro-de-energia-sostiene-que-esnecesario-un-ajuste-en-las-tarifas-de-gas-en-magallanes.html 
incluyó a dirigentes sociales de la zona, al gobierno y al Obispo Bastres, ${ }^{6}$ quien medió en el conflicto. ${ }^{7}$ Adicionalmente, las críticas a la conducción política de este conflicto fueron uno de los elementos que gatillaron el primer cambio de gabinete ocurrido el 16 de enero. El 18 de enero se anunció que el gobierno y los habitantes de Magallanes habían llegado a acuerdo, poniendo así término al conflicto. ${ }^{8}$ Este incluyó un alza del precio del gas de sólo 3\%, la entrega de 15 mil subsidios adicionales y el compromiso, por parte del gobierno, de enviar un proyecto de ley que establezca los criterios para definir el precio del combustible en la región. ${ }^{9}$

El segundo movimiento de tipo local se produjo en Calama, Región de Antofagasta, durante los meses de junio y agosto. La primera jornada de paralización tuvo lugar el 29 de junio, bajo la consigna "producimos cobre, seguimos pobres", en la cual se denunciaba el abandono de las autoridades y se demandaba dejar el $5 \%$ de las utilidades del cobre en la ciudad. ${ }^{10}$ La segunda jornada de paro de la ciudad se realizó dos meses después, el 29 de agosto. En esa oportunidad, los organizadores cifraron en 18.000 personas los asistentes al acto, el cual dejó como saldo un carabinero herido y al alcalde de la comuna, detenido. ${ }^{11}$ El conflicto se distendió con la constitución de una mesa de trabajo que entregaría sus conclusiones en marzo de 2012.

Las movilizaciones se desarrollaron también durante 2011 en torno a temas asociados al cuidado del medio ambiente, destacando especialmente las que se produjeron a raíz de la aprobación del proyecto HidroAysén, que pretende construir cinco represas en los ríos Baker y Pascua. El proyecto fue aprobado por la Comisión de Evaluación Ambiental en Coyhaique el 9 de mayo, ${ }^{12}$ gatillando múltiples marchas y actos públicos contra el proyecto en distintas ciudades del país, las que se mantuvieron con fuerza durante mayo y comienzos de junio. El gobierno defendió el proyecto y señalaba que éste había sido aprobado conforme a los canales institucionales de la toma de decisión. ${ }^{13}$ Con todo, no se descarta que el conflicto pueda volver a surgir, ya que aún no se resuelve definitivamente sobre el destino del proyecto.

La fuerza que aparentaba tener la movilización en temas medioambientales fue, sin embargo, opacada por el inicio de las actividades de protesta del movimiento estudiantil,

6 Ya en 2010 había sido necesaria la mediación de altas autoridades de la Iglesia Católica, cuando el entonces obispo Ezzati intermedió en las negociaciones llevadas a cabo para terminar con la huelga de hambre sostenida por mapuches.

7 http://www.emol.com/noticias/nacional/2011/01/12/458103/gobierno-anuncia-inicio-de-conversacionesen-magallanes-con-obispo-bastres-como-facilitador.html

8 http://www.emol.com/noticias/nacional/2011/01/18/459162/gobierno-y-habitantes-de-magallanes-sellanacuerdo-que-pone-fin-al-conflicto-del-gas.html

9 El proyecto fue ingresado al Congreso en septiembre.

10 http://www.emol.com/noticias/nacional/2011/06/29/489810/habitantes-de-calama-inician-paro-enprotesta-por-abandono-de-las-autoridades.html

11 http://www.emol.com/noticias/nacional/2011/08/29/500439/organizadores-cifran-en-18-mil-los-asistentesa-acto-por-paro-comunal-en-calama.html

12 http://www.emol.com/noticias/nacional/2011/05/09/480625/manifestaciones-marcan-jornada-deaprobacion-del-proyecto-hidroaysen.html

13 http://www.emol.com/noticias/nacional/2011/05/21/482851/pinera-realiza-ferrea-defensa-de-hidroaysendurante-cuenta-publica-ante-el-congreso.html 
liderado por la Confederación de Estudiantes de Chile $(\mathrm{CONFECH})$, instancia que reúne a las federaciones de estudiantes de las universidades tradicionales del país más algunas privadas. La actividad del movimiento estudiantil se inició en mayo, transformándose en el más importante de su tipo en los últimos años, ${ }^{14}$ y en el principal problema político del gobierno de Piñera. En su discurso del 21 de mayo de 2011, Piñera anunció que este sería el "año de la educación superior", indicando que a las reformas a la calidad de la educación aprobadas en febrero de 2011 y a la creación de la Agencia de Calidad y Superintendencia de Educación Escolar, se sumarían nuevas tareas para el año (Piñera, 2011). En ese marco, bajo el liderazgo de la CONFECH, y en menor medida por las organizaciones de estudiantes secundarios, los estudiantes comenzaron a desplegar una intensa movilización dirigida a presentar y demandar al gobierno un conjunto de reformas al sistema educacional chileno. En concreto, la misma CONFECH definió como sus objetivos inmediatos los siguientes: "educación gratuita, fin al lucro, democratización, fin al endeudamiento y el autofinanciamiento y acceso equitativo" (CONFECH, 2011: 1). Para el efecto, el movimiento utilizó diversas formas de movilización, como las tomas de los establecimientos educacionales (algunas de las cuales se prolongaron por más de seis meses), paros, marchas masivas, huelgas de hambre, y llamados a protestas masivas a través de "cacerolazos".

Ante el rechazo a las demandas expresado por el entonces ministro de Educación, Joaquín Lavín (UDI), el movimiento estudiantil adquirió fuerza creciente, y un gran apoyo en la población, que se expresó en distintas encuestas de opinión (www.adimark.cl) y también en una amplia participación en las marchas que se convocaron. Ante esto, en primer lugar el gobierno atinó a plantear un proyecto de reforma educacional conocido como GANE, con fuerte énfasis en la inyección de recursos al sistema, pero sin modificar sustantivamente la estructura educacional del país. ${ }^{15}$ Esto fue rechazado inmediatamente por el movimiento, lo que contribuyó a la salida de Lavín del ministerio, junto con la existencia de un problema de conflicto de interés no resuelto por parte del ministro. Su sucesor, Felipe Bulnes (RN), buscó hacer frente al problema a través de distintas propuestas, ${ }^{16}$ que una y otra vez fueron rechazadas, lo que determinó que el conflicto se prolongara, en términos tales que varias universidades y liceos no funcionaron por más de cinco meses. Hacia fines de año, y en el marco de la discusión de la ley de presupuestos, el gobierno buscó desactivar el conflicto por medio de la inyección de nuevos recursos al sistema y la promesa de que en 2012 se seguiría ocupando del tema. Al mismo tiempo, y debido a diversos factores (decisión de volver a clases, término de tomas, y recambio de liderazgos en las federaciones universitarias), se observó una baja en la intensidad de la movilización estudiantil. De esta forma, hacia fines de año el sistema educacional volvió a funcionar en orden a cerrar como se pudiera el año escolar, mientras el gobierno argumentaba que la fase más difícil había sido superada. Con

14 Pero no el único. Destaca, por ejemplo, la denominada "revolución pingüina" de 2006. (ver Mardones, 2007).

15 http://www.emol.com/noticias/nacional/2011/07/05/490990/presidente-pinera-convoca-a-un-granacuerdo-nacional-por-la-educacion.html

16 Ver, por ejemplo: http://www.biobiochile.cl/2011/08/17/ministro-bulnes-entrega-nuevas-propuestas-parala-educacion.shtml 
todo, es difícil sostener que el problema se encuentre resuelto. Los estudiantes siguen sosteniendo que en 2012 volverán a presionar al gobierno para que éste implemente las reformas por ellos solicitadas (que aún están lejos de cumplirse). Igualmente, el ministro Bulnes renunció a su cargo a fines de 2011, designándose en su reemplazo a un experto en temas educacionales, lo que refleja que el gobierno se prepara también para un año difícil en la materia.

Más allá de lo que significó poner el tema educacional al centro de la agenda pública, el caso del movimiento estudiantil de 2011 fue muy significativo en términos políticos, desde varias perspectivas. En primer lugar, puso de manifiesto la incapacidad del gobierno para lograr una salida relativamente rápida y favorable para sus intereses. Por el contrario, el resultado fue que el conflicto se mantuvo por más de seis meses (cayendo dos ministros), y sin que hasta la fecha se haya resuelto. Más bien se encuentra encapsulado durante las vacaciones de verano (diciembre-marzo). Además, en el contexto del conflicto los niveles de aprobación de Piñera cayeron a mínimos históricos (comparando los cinco gobiernos desde 1990).

En segundo lugar, los partidos políticos de ambas coaliciones mostraron nula capacidad para encauzar el conflicto, al igual que en el caso de las movilizaciones locales. Más aún, a lo largo del año los estudiantes repetidamente manifestaron su rechazo tanto a la Coalición por el Cambio como a la Concertación, despreciando el trabajo conjunto con ellos. ${ }^{17}$ Desde el punto de vista de los partidos de la Concertación, el conflicto estudiantil representó una oportunidad para lograr rearticular su discurso y acción política conjunta. Sin embargo, ya sea por la defensa de logros anteriores, por el rechazo a lo obrado en los gobiernos anteriores, o por la falta de liderazgos claros, la Concertación no logró definir una postura clara y única en torno al conflicto. En la práctica, el conflicto estudiantil puso en evidencia su debilidad y el conflicto interno que ha sido evidente desde la pérdida de las elecciones de 2009/2010.

En tercer lugar, si bien el movimiento estudiantil ha sido exitoso en términos de redefinir la agenda pública, hasta el momento no ha sido capaz de que las decisiones públicas realicen sus demandas. En este sentido, es probable que su rechazo a la colaboración con los partidos juegue en contra de sus intereses (después de todo, cualquier reforma legal que materialice sus demandas requiere el voto de los parlamentarios). Asimismo, y aun cuando no sea directamente atribuible al movimiento, la violencia observada al terminar la mayoría de las marchas por la educación supone una incapacidad del movimiento para controlarla y con ello abrir un flanco para debilitarlo, como efectivamente lo intentó el gobierno.

En cuarto lugar, el movimiento cambió la agenda pública para el 2012, ya que abrió la puerta a la discusión de una reforma tributaria que permitiría financiar una gran reforma educacional, a la vez que dio impulso a una nueva discusión sobre la necesidad de reformar el sistema electoral binominal.

17 Esto, tal vez, con la excepción parcial del PC, partido al que pertenecen dos importantes líderes del movimiento. Con todo, no se sabe si esto le va a beneficiar política y electoralmente en el futuro. 
Más allá de los conflictos sociales, en el primer semestre de 2011 tuvieron lugar dos episodios que afectaron negativamente la imagen del gobierno y sus partidos, y donde estos demostraron poca capacidad para resolverlos positivamente. El primero se refiere a la salida de su cargo de la ex intendenta de la Región del Bío-Bío, Jacqueline van Rysselberghe (UDI). En febrero, un senador de oposición denunció que la intendenta había "inventado" un proyecto con el objeto de que habitantes de un sector de la ciudad (la Población Aurora), que no habían sido afectados significativamente por el terremoto de 2010, accedieran a beneficios contemplados para quienes efectivamente lo habían sido. ${ }^{18}$ Esta denuncia desató una dura pugna entre gobierno y oposición, la que pronto anunció que acusaría constitucionalmente a la intendenta para así removerla del cargo. La UDI reaccionó desplegando una fuerte presión sobre el gobierno para que no la destituyera, la que en principio tuvo éxito pues el gobierno rechazó la acusación (aun cuando señaló que se había equivocado). ${ }^{19}$ Sin embargo, y en lo que demostró la incapacidad del gobierno y sus partidos de actuar en forma coordinada, con el avance del tiempo fue quedando claro que en RN había parlamentarios dispuestos a apoyar la acusación, la que fue presentada en marzo. ${ }^{20} \mathrm{Y}$ esto a pesar de los esfuerzos del gobierno y dirigentes de la UDI por "ordenar" a RN. Estando claro que el escenario más probable era la aprobación de la acusación en la Cámara de Diputados, van Rysselberghe optó por renunciar a su cargo, ya que en definitiva (dada la mayoría opositora en el Senado) su destitución era lo más probable.

El segundo tuvo que ver con la salida de su cargo de la ministra de Vivienda, Magdalena Matte. Si bien ella había sido cuestionada durante el caso de van Rysselberghe (cuya actuación Matte rechazó desde un principio), su salida se produjo en las semanas siguientes como consecuencia del denominado "caso Kodama". A principios de abril se hizo público que el ministerio había llegado a un acuerdo extrajudicial con la empresa constructora Kodama, que a fines de 2010 había demandado al fisco solicitando el pago de cerca de 35 millones de dólares por haber incurrido en gastos extraordinarios en la construcción de un corredor de buses en Santiago. ${ }^{21} \mathrm{El}$ acuerdo, que se firmó a poco más de un mes de presentada la demanda, fue negociado por un cercano asesor de la ministra, que desde hace mucho tiempo era su abogado de confianza. La ministra se defendió señalando que, si bien ella había firmado el decreto autorizando el pago, éste no se produjo y que desde un principio entregó la información a quienes correspondía. Esta vez, y a diferencia del caso anterior, la ministra recibió un apoyo cerrado por parte de RN y la UDI. Sin embargo, ello no fue suficiente, pues inmediatamente se inició un proceso judicial para investigar los eventuales delitos que podrían haberse cometido, lo

18 http://www.emol.com/noticias/nacional/2011/02/03/462371/denuncian-que-van-rysselberghe-inventohistoria-para-lograr-subsidios-habitacionales.html

19 http://www.emol.com/noticias/nacional/2011/02/10/463750/udi-arremete-contra-navarro-y-pide-algobierno-respaldar-a-la-brevedad-a-intendenta.html

20 http://www.emol.com/noticias/nacional/2011/03/23/471758/lider-de-rn-y-caso-van-rysselberghe-paraque-insistir-en-una-autoridad-tan-cuestionada.html

21 http://www.emol.com/noticias/nacional/2011/04/14/475995/ministra-matte-los-que-tienen-responsabilidadestienen-que-asumirla.html 
que terminó por precipitar la renuncia de la ministra, y el descabezamiento de la plana mayor del ministerio. ${ }^{22}$ Hoy, la investigación sigue en curso.

\section{b) Coyuntura económica}

Pese a la crisis económica europea vivida durante el 2011, y que se proyecta hacia el 2012, el desempeño económico de Chile ha sido positivo durante este período, aunque muestra signos de desaceleración para el tiempo próximo. En efecto, las cifras de crecimiento económico (con respecto al mismo trimestre del año anterior) muestran que Chile creció con fuerza los dos primeros trimestres del año (9,9\% y 6,6\% respectivamente), mientras que el crecimiento el tercer trimestre se redujo al 4,8\% (Corbo, 2012). El empleo se ha mantenido relativamente alto, con una tasa de desocupación del 6,6\% para el trimestre móvil de octubre-diciembre de 2011 y un promedio del 7,1\% para el 2011, lo cual representa una caída en el desempleo de 0,5 puntos porcentuales en doce meses (INE, 2012). La inflación por su parte, muestra una variación del $4,4 \%$ para el año, relativamente superior a las expectativas iniciales, dado especialmente por la variación de $0,6 \%$ que se registró en diciembre (INE, 2012). Pese a estos niveles relativamente positivos de inflación general, es importante destacar que algunas de las alzas más importantes registradas durante el año se dieron en áreas particularmente sensibles para las personas, como las alzas en transporte, electricidad, suministro de agua y entre los alimentos y bebidas no alcohólicas (INE, 2012).

A nivel de empresas, la coyuntura estuvo marcada por el escándalo de La Polar, empresa del retail, que dejó en evidencia el problema de los abusos del sistema económico con sus consumidores. El caso estalló el 2 de junio, con la presentación por parte del Servicio Nacional del Consumidor (SERNAC) de una demanda colectiva contra la empresa por haber repactado unilateral e ilegalmente las deudas de sus clientes. ${ }^{23}$ La magnitud del caso fue conocida durante los meses siguientes, llegando a establecerse que más de un millón de clientes habían sido afectados por esta práctica. ${ }^{24}$ A nivel de la empresa este caso tuvo repercusiones en diversas áreas, como el cambio en sus ejecutivos y miembros del directorio, la caída en el valor de las acciones, y en la implementación de planes de compensación para los clientes. A nivel judicial, una serie de acciones legales emprendidas por diversos actores ha llevado al avance de la investigación por las responsabilidades -tanto penales como civiles- de sus ejecutivos. En el hecho más significativo, el 14 de diciembre fueron formalizados tres altos ejecutivos por cargos que incluyen el lavado de dinero, uso de información privilegiada, entrega de información falsa al mercado, entre otros. Como resultado, los tres ex ejecutivos están en prisión preventiva y el caso continúa en tramitación. ${ }^{25}$ A nivel político, el desarrollo de este escándalo llevó a acelerar la tramitación del proyecto de ley que crea el "SERNAC Financiero". El proyecto, que

http:/ /www.emol.com/noticias/nacional/2011/04/19/476900/ministra-matte-presenta-su-renuncia-enmedio-de-polemica-por-caso-kodama.html

23 http://www.emol.com/noticias/economia/2011/06/02/485091/sernac-presenta-demanda-colectiva-contrala-polar-por-repactaciones-unilaterales.html

24 http://www.emol.com/noticias/economia/2011/08/17/498299/pinera-un-millon-de-personas-fueronabusadas-por-la-polar-y-eso-no-lo-vamos-a-permitir.html

25 http://www.emol.com/noticias/economia/2011/12/16/517332/xxx.html 
había iniciado su tramitación en enero de 2011, establece nuevas regulaciones al sistema financiero y busca una mayor protección de los consumidores en estas materias. La ley entrará en vigencia en marzo de 2012.

\section{c) Coyuntura subregional e internacional}

La llegada a la presidencia de Piñera en 2010 no significó un cambio sustantivo de las líneas directrices de la política exterior. En ese contexto, la estrategia internacional del nuevo gobierno se ha caracterizado por una profunda continuidad con la de sus antecesores, en términos de que sigue guiada por las mismas prioridades y conflictos.

En el ámbito subregional, la agenda chilena se ha concentrado en las relaciones con los países vecinos. En el caso de Perú, el elemento que sigue marcando la relación entre los países es el juicio que se sigue en La Haya, originado en una petición peruana de revisar los límites entre los países (Mardones, 2008). En 2011 concluyó la fase escrita del pleito, esperándose para 2012 la realización de la fase oral, aun cuando es improbable que el tribunal emita su decisión este año. No obstante esta situación, el gobierno ha insistido en su posición de que el diferendo limítrofe no puede bloquear el desarrollo de otros aspectos de la relación, ya que Perú sigue siendo un país muy relevante para Chile (por ejemplo, es uno de los principales destinos de la inversión extranjera chilena). En ese marco, el gobierno sigue apostando al desarrollo de distintas iniciativas conjuntas con Perú, vinculadas al control de fronteras o la integración energética (Moreno, 2011).

En el caso de Bolivia, a partir de 2010 el gobierno optó por darle continuidad al esquema de relación con Bolivia desarrollado por Bachelet, que tenía como marco la denominada "Agenda de 13 puntos". Sin embargo, a comienzos de 2011 la relación bilateral se estancó, luego de que el presidente Morales anunciara que demandaría a Chile en La Haya en orden a obtener a una salida al mar para su país, ${ }^{26}$ lo cual hasta ahora no se ha materializado, aun cuando sí trató de plantear este punto en instancias multilaterales. El gobierno, como sus antecesores, rechazó la actitud boliviana insistiendo que el tema marítimo está zanjado por el Tratado de 1904, y que las diferencias entre los países no se deben judicializar, tampoco llevar a discusión en órganos multilaterales.

Por último, en el caso de Argentina el progreso de las relaciones ha sido más favorable, ya que ambos países se han concentrado en la implementación del "Tratado de Maipú" de 2009, marco en el cual se han implementado distintos mecanismos para fortalecer la integración física entre los países, como los comités birregionales y la eventual construcción de nuevos túneles a través de la cordillera. Con todo, persiste entre los países el conflicto derivado de la decisión del gobierno de Cristina Fernández de darle a Galvarino Apablaza (cuya extradición fue solicitada por Chile por su participación en el homicidio del líder UDI, Jaime Guzmán) la calidad de refugiado político. Chile rechazó esa decisión, pero antes de provocar un conflicto fuerte con Argentina, optó por seguir las vías administrativas en orden a obtener la extradición.

26 http://www.emol.com/noticias/internacional/2011/03/23/471776/bolivia-llevara-a-chile-ante-la-justiciainternacional-por-salida-al-mar.html 
Más allá de las relaciones con los vecinos, en el ámbito regional Chile ha seguido desarrollando su estrategia de "regionalismo abierto". En este marco, una de sus prioridades ha sido el impulso de la "Alianza del Pacífico", junto a Colombia, México y Perú, la cual apunta precisamente a profundizar la relación comercial entre los países, en distintas materias como bienes, inversiones, mercado de capitales y reglas de origen, además de desarrollar una estrategia conjunta de vinculación con la región Asia-Pacífico. En este sentido, fue relevante también la suscripción de un "Acuerdo de Asociación Estratégica" con Colombia. Además, en el plano sudamericano, Chile ha seguido con su política de participar activamente en los esquemas de concertación política entre los países de América Latina, destacando al efecto, el haber asumido para 2012 la presidencia de la Comunidad de Estados Latinoamericanos y Caribeños (CELAC), entidad sucesora del Grupo de Río. ${ }^{27}$

Fuera del ámbito propiamente latinoamericano, Chile ha apostado a reforzar las ya buenas relaciones con los "polos de desarrollo", como Estados Unidos y Europa. Igualmente, y en el marco de la mantención de la estrategia de regionalismo abierto, se firmaron tratados de libre comercio con Vietnam y Malasia, además de iniciarse las negociaciones para concretar un tratado comercial con Hong Kong. En este mismo contexto, Chile ha sido impulsor principal de la Trans Pacific Partnership (TPP), que busca crear la zona de libre comercio más grande del mundo (Moreno, 2011).

\section{Cambios institucionales y cambios sustanciales en políticas públicas}

La aprobación de la ley que hace efectivo el voto voluntario y establece la inscripción automática de todos los ciudadanos con derecho a voto fue una reforma institucional fundamental. ${ }^{28}$ Esta ley, que fue promulgada en enero de 2012, permitirá que los cambios al sistema de inscripción y voto se hagan efectivos en la elección municipal de 2012, lo cual supone un cambio de escenario fundamental: más de 4.000 .000 de nuevos inscritos tendrá el renovado padrón electoral. La gran mayoría de estos nuevos inscritos, además, tienen entre 18 y 30 años de edad, rejuveneciendo de manera significativa al electorado. Al mismo tiempo, sin embargo, la ley cambia el carácter del voto de uno obligatorio a otro voluntario. En su conjunto, estas modificaciones introducen fuertes incertidumbres de cara a las próximas elecciones: primero, el contenido y forma de las campañas electorales podría cambiar, dada la ampliación en el número y tipo de votantes; segundo, no hay claridad respecto de si estos nuevos electores ejercerán o no su derecho a voto y si aquellos que ya votaban con anterioridad lo seguirán haciendo; $\mathrm{y}$, tercero, se generan incertidumbres respecto de la estabilidad o cambio que podrían observarse en las preferencias políticas agregadas de la población.

En relación a la estructura organizacional del Estado central, hay dos cambios a destacar. Primero, el 21 de febrero entró en vigencia la Ley No 20.502 que creó el nuevo Ministerio

28 Con ella se materializó la reforma constitucional de 2009 que estableció el voto voluntario pero que, para entrar en vigencia, requería de una modificación legal. 
del Interior y Seguridad Pública ${ }^{29}$ luego de cuatro años de tramitación. Con esto, Interior asume mayor control de las tareas de prevención y control de la delincuencia, así como tareas de reinserción y rehabilitación social de los infractores. La ley traspasa también a la Subsecretaría del Interior el control de Carabineros y la Policía de Investigaciones (PDI), que antes eran dependientes del Ministerio de Defensa. Segundo, el 13 de octubre se publicó la Ley $\mathrm{N}^{\mathrm{o}} 20.530$ que creó el Ministerio de Desarrollo Social, ${ }^{30}$ que reemplaza al Ministerio de Planificación (MIDEPLAN). El proyecto de ley, que había ingresado al Congreso en agosto de 2010, era parte de los anuncios del Presidente del 21 de mayo de 2010. ${ }^{31}$ El nuevo ministerio tiene como funciones principales la articulación de los distintos programas sociales del ministerio, la coordinación con otros ministerios en materias de protección social, y la evaluación y control de los programas existentes. ${ }^{32}$

En materias de políticas públicas, durante el 2011 se aprobaron dos leyes importantes: la que estableció un período de postnatal de seis meses y la que eliminó la cotización del 7\% en salud para los jubilados. Contar con una ley de postnatal ${ }^{33}$ mejorado y más extenso había sido una de las promesas de la campaña presidencial de Piñera. La tramitación y debate de este proyecto, pese a contar con apoyo en todos los sectores políticos, fue muy compleja y en sus primeras etapas bastante desordenada, concentrándose las discrepancias en torno a la extensión del beneficio. Con todo, la ley fue finalmente promulgada el 6 de octubre (Ley $\left.\mathrm{N}^{\mathrm{0}} 20.545\right) .{ }^{34}$ En concreto, se estableció un período de postnatal de seis meses para todas las mujeres, cubriendo el sueldo de ellas con un tope de 66 U.F. mensuales -alrededor de tres mil dólares. En lo esencial, se dispone que son elegibles para obtener el beneficio no sólo las mujeres con contrato formal de trabajo, sino que también las trabajadoras independientes y aquellas con contratos por obra o faena. Los tres primeros meses del permiso de maternidad son obligatorios y deben ser tomados en su totalidad. Los tres meses siguientes pueden ser utilizados de dos maneras: o se toman los tres meses, con permiso laboral por jornada completa, o se puede volver al trabajo, en media jornada por un período de seis meses.

El 29 de agosto fue promulgada la Ley $\mathrm{N}^{\mathrm{o}} 20.531^{35}$ que exime o reduce la cotización del $7 \%$ en salud a los pensionados. ${ }^{36}$ La norma establece que los pensionados que son parte del Sistema de Pensiones Solidario quedan exentos de pagar el 7\% de cotización para salud. Adicionalmente, todos los pensionados de 65 años o más que no pertenecen al pilar solidario, pero que sí se ubican dentro de los cuatro quintiles de menores ingresos se beneficiarán de una reducción de su cotización del 7\% al 5\% (Larraín, 2011).

http:/ / www.leychile.cl/Navegar?idLey=20502\&idVersion=2011-02-21

http:/ / www.leychile.cl/Navegar?idLey=20530\&idVersion=2011-10-13

http:/ /www.gobiernodechile.cl/media/2010/05/Mensaje-presidencial-21-de-mayo.pdf

http://www.emol.com/noticias/nacional/2011/10/13/507838/promulgacion-ministerio-de-desarrollo-social.html

Período de permiso del trabajo pagado, que en el caso de las mujeres se entrega antes y después del nacimiento de un hijo/a.

http: / /www.leychile.cl/Navegar?idLey=20545\&idVersion=2011-10-17

http:/ /www.leychile.cl/Navegar?idNorma=1028994

http:/ / www.emol.com/noticias/nacional/2011/08/28/500208/gobierno-promulgo-ley-que-elimina-el-7-

de-cotizacion-a-los-jubilados.html 


\section{Poder Ejecutivo}

Durante 2011 se produjeron cuatro cambios de gabinete. La apuesta del Presidente al comienzo de su mandato fue nombrar un gabinete de expertos compuesto por destacadas personalidades del mundo de la empresa y la academia, más que del mundo de la política. La idea era tener el gabinete "de los mejores". La Tabla 1 muestra la composición original del gabinete que asumió en marzo de 2010. Tal como se aprecia, de los 22 ministerios, 4 correspondieron a militantes de RN y 4 a la UDI. Los 14 ministros restantes no tenían filiación política formal. Entre ellos, destaca el caso de Jaime Ravinet, quien había sido ministro de Lagos, pero que renunció a su militancia en el Partido Demócrata Cristiano antes de asumir en su cargo. Por otra parte, 6 de los ministros $(23 \%)$ eran mujeres.

Los sucesivos cambios de gabinete reflejaron la necesidad de superar el problema de tener ministros sin experiencia política. La Tabla 2 muestra los cambios producidos durante 2011. Con respecto a estos cambios destaca lo siguiente. Primero, algunos de los cambios producidos durante el año son "enroques" entre ministerios: ministros que salen de uno para entrar a otro. Un ejemplo es el caso de Laurence Golborne quien durante el primer cambio de gabinete, producido el 16 de enero, asumió como biministro de Energía y Minería y, en el segundo cambio producido en julio, deja ambos ministerios para asumir como ministro de Obras Públicas.

Segundo, tanto en el primer como en el segundo cambio de gabinete, Piñera decidió nombrar como ministros a miembros en ejercicio del Senado, precisamente con el objeto de darle "más capacidad política" a su gobierno. Al respecto, cabe mencionar que, aunque ajustados a derecho, estos nombramientos y las vacancias consecuentes dejadas en el Senado provocaron una importante discusión, ya que a través de ellos se privaba a los electores de sus representantes.

Tercero, varios cambios fueron producto de los problemas que el gobierno enfrentó durante el año. Así, por ejemplo, se reemplazó al ministro Rainieri -de Energía- en medio de las manifestaciones de Magallanes por el alza del gas. Magdalena Matte salió en medio de los cuestionamientos del Ministerio de Vivienda por el caso Kodama, además de que los dos cambios en el Ministerio de Educación tuvieron que ver con el fracaso de la estrategia del gobierno para resolver el conflicto.

Finalmente, los casos de Fernando Echeverría y Luis Mayol merecen mención especial. Echeverría había asumido al comienzo del gobierno como Intendente de Santiago. El 18 de julio había jurado como nuevo ministro de Energía y debió renunciar dos días después, señalando que podrían haber "conflictos de interés" del cargo con los negocios en los que había participado. Esto obligó a un nuevo nombramiento en la cartera el 22 de julio. Este episodio reveló dos aspectos importantes. Por una parte, el problema de conflictos de interés que había estado presente durante todo el año 2010 y, por la otra, los problemas de gestión enfrentados por el gobierno. ${ }^{37}$ Con respecto a Luis Mayol, cabe 


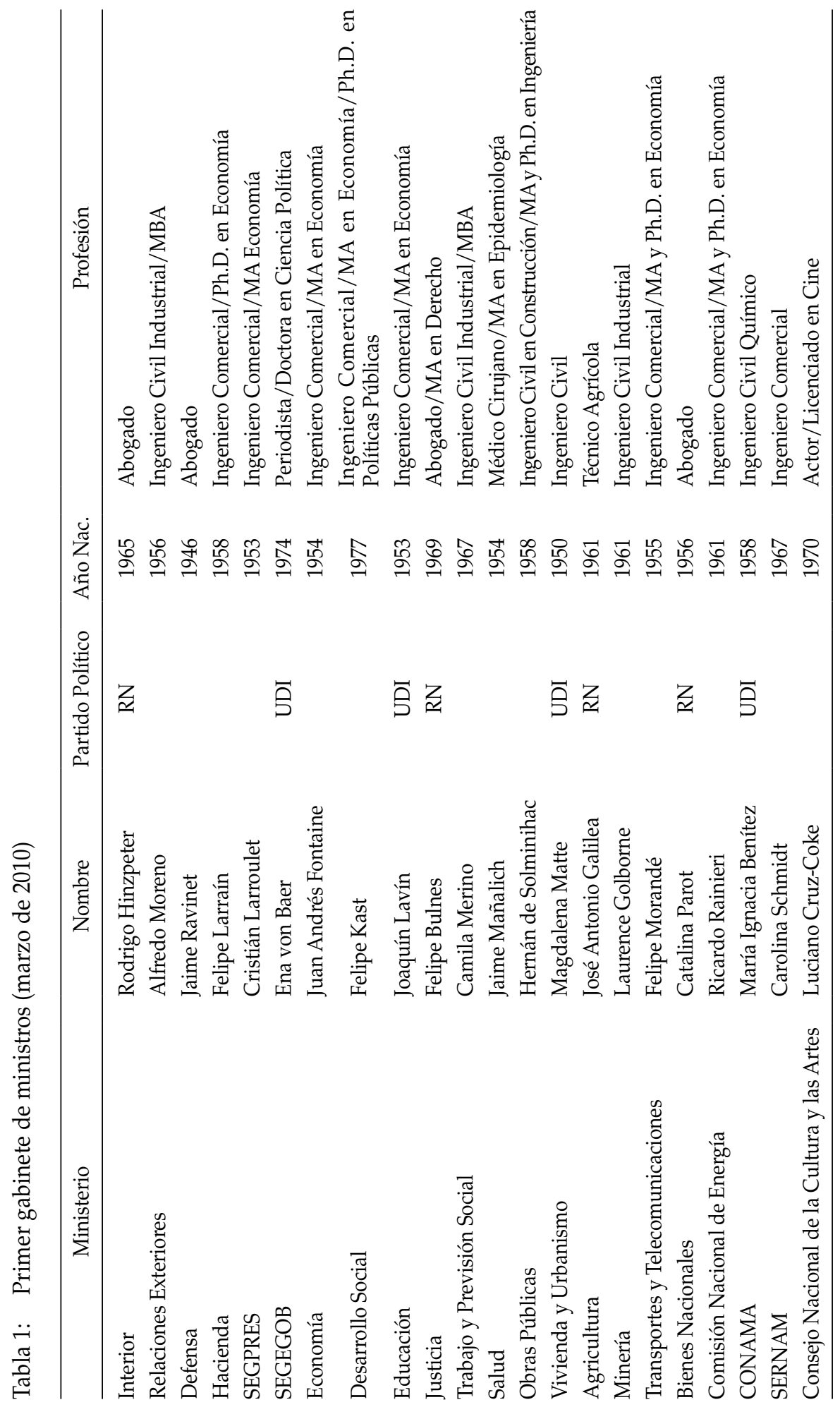




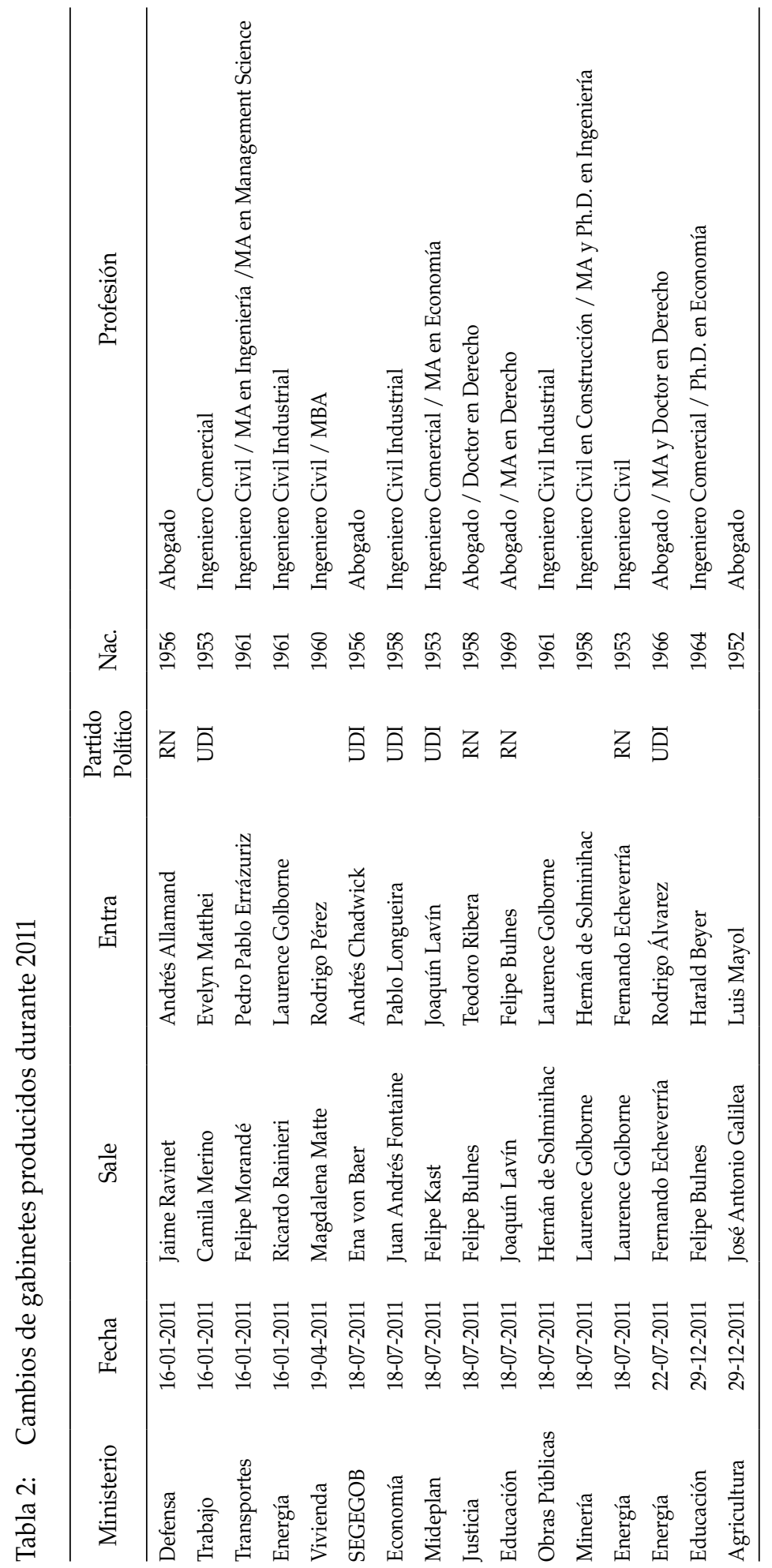


resaltar que al momento de su nombramiento se desempeñaba como presidente de la Sociedad Nacional de Agricultura (SNA), la organización gremial que reúne a los grandes empresarios del sector agrícola: desde 1990 no había ocurrido que un alto dirigente gremial asumiera la dirección de la política gubernamental en la materia de su interés.

\section{Poder Legislativo}

El Poder Legislativo experimentó durante 2011 una serie de modificaciones en su composición. Las Tablas 3 y 4 describen la composición partidaria de la Cámara de Diputados y del Senado en marzo de 2010 y diciembre de 2011. Estas muestran, por una parte, algunas alteraciones en la distribución de escaños por partidos en la Cámara de Diputados debido a la renuncia de algunos parlamentarios a los partidos que los eligieron.

Tabla 3: Conformación de la Cámara de Diputados (2010-2014)

\begin{tabular}{lcc}
\hline \multicolumn{1}{c}{ Partido } & Marzo de 2010 & Diciembre de 2011 \\
\hline Unión Demócrata Independiente & 39 & 39 \\
Renovación Nacional & 18 & 17 \\
Partido Demócrata Cristiano & 19 & 19 \\
Partido por la Democracia & 19 & 18 \\
Partido Socialista & 11 & 11 \\
Partido Radical Socialdemócrata & 5 & 5 \\
Partido Comunista & 3 & 3 \\
Partido Regionalista Independiente & 2 & 2 \\
Independientes & 4 & 6 \\
\hline Total & 120 & 120 \\
\hline
\end{tabular}

Tabla 4: Conformación del Senado (2010-2014)

\begin{tabular}{lcc}
\hline \multicolumn{1}{c}{ Partido } & Marzo de 2010 & Diciembre de 2011 \\
\hline Unión Demócrata Independiente & 8 & 8 \\
Renovación Nacional & 8 & 8 \\
Partido Demócrata Cristiano & 9 & 9 \\
Partido por la Democracia & 4 & 4 \\
Partido Socialista & 5 & 5 \\
Partido Radical Socialdemócrata & 1 & 1 \\
Movimiento Amplio al Socialismo & 1 & 1 \\
Independientes & 2 & 2 \\
\hline Total & 38 & 38 \\
\hline
\end{tabular}


Por otra parte, y pese a no alterar los equilibrios partidarios, se produjeron una serie de reemplazos de parlamentarios. Esto con el objeto de llenar las vacantes generadas por los senadores que han pasado a integrar el Ejecutivo (4), los diputados que han pasado al Senado (2), además de la generada por la muerte de un diputado (Juan Lobos, UDI).

Respecto de la actividad legislativa durante el 2011, ya se han comentado algunas de las leyes que terminaron su tramitación durante el año. La siguiente Tabla muestra algunas estadísticas oficiales respecto de la labor realizada en el Congreso durante 2010 y 2011. A este respecto, cabe subrayar dos puntos relacionados. Primero, que al igual que en los últimos 22 años, el hecho de que la coalición gobernante no cuente con mayoría en el Congreso, no se ha traducido en una situación de bloqueo legislativo, permitiendo al Ejecutivo hacer avanzar sus proyectos sin dificultades mayores. Segundo, esto contradice el discurso oficialista de que la oposición parlamentaria ha sido fundamentalmente obstruccionista, ya que si así fuera la producción legislativa sería sustantivamente menor. Incluso, este supuesto obstruccionismo es desmentido por el ministro Larroulet quien, al presentar su cuenta pública 2011 señala que "logramos este año aprobar el mayor número de leyes de la última década" ${ }^{38}$

\section{Relación entre los poderes del Estado}

A este respecto, aparte de las diferencias entre la oposición y el gobierno que significaron distintos conflictos entre Ejecutivo y Legislativo, destaca el que la última parte del 2011 estuvo marcada por una dura disputa entre el Ministerio del Interior, y el Poder Judicial y el Ministerio Público (MP). El tema de la seguridad pública había sido central en la campaña electoral de 2009/2010, donde la candidatura de Piñera había prometido una

Tabla 5: $\quad$ Estadísticas labor legislativa, 2010-2011

\begin{tabular}{lcccc}
\hline \multirow{2}{*}{ Total proyectos ingresados } & \multicolumn{2}{c}{2011} & \multicolumn{2}{c}{2010} \\
\cline { 2 - 5 } & $\mathrm{N}$ & $\%$ & $\mathrm{~N}$ & $\%$ \\
\cline { 2 - 5 } & 728 & 100 & 588 & 100 \\
\hline Moción & 621 & 85,3 & 486 & 82,7 \\
Mensaje & 107 & 14,7 & 102 & 17,3 \\
\hline Total leyes publicadas en el Diario Oficial & 87 & 100 & 96 & 100 \\
Moción & 30 & 34,5 & 31 & 32,3 \\
Mensaje & 57 & 65,5 & 65 & 67,7 \\
\hline
\end{tabular}

Fuente: Cámara de Diputados, Resumen Estadístico 2010 y 2011. Las estadísticas consideran el período que va desde el 1 de enero al 31 de diciembre de cada año.

38 http://www.emol.com/noticias/nacional/2011/12/23/518469/ministro-larroulet-logramos-este-ano-aprobarel-mayor-numero-de-leyes-de-la-ultima-decada.html 
lucha frontal contra la delincuencia ("a los delincuentes se les acabó la fiesta", decía Piñera).

No obstante en abril de 2011 el ministro del Interior, Rodrigo Hinzpeter (RN), había hecho un muy positivo balance de la gestión del gobierno en la materia, ${ }^{39}$ el escenario cambió sustantivamente a inicios de octubre. En ese momento, y como respuesta a los actos de violencia que se habían producido recurrentemente en el marco de las movilizaciones por la reforma educacional y que la autoridad no podía controlar, el gobierno envió un proyecto de ley que aumenta las penas para quienes participaran en saqueos y penaliza las tomas de inmuebles (p.e. de las universidades) y las interrupciones del tránsito. Al justificar el envío del proyecto, el gobierno criticó duramente a las autoridades judiciales, ya que a su juicio quienes habían participado en actos delictuales con ocasión de las manifestaciones no recibían las sanciones que merecían, y rápidamente obtenían su libertad. ${ }^{40}$ Si bien esto fue firmemente rechazado desde un principio por la Corte Suprema, como también por la Asociación de Magistrados, ${ }^{41}$ el conflicto escaló fuertemente luego de que el ministro de Justicia, Teodoro Ribera (RN), insinuara que el Ejecutivo (que de acuerdo a la Constitución es el encargado de designar a los jueces, a proposición de las Cortes) evaluaría el ascenso de los jueces sobre la base de sus fallos. ${ }^{42}$ Ante esto, la Corte Suprema manifestó su enérgico rechazo a estas declaraciones, recordándole al gobierno que se trata de un poder autónomo y advirtiéndole que siempre velarán por la independencia de los jueces. La Asociación de Magistrados calificó esto como una "intimidación". ${ }^{43}$ Ante esto, el gobierno prefirió no escalar el conflicto, aun cuando no cesó en solicitar que los jueces sean "más duros" con los delincuentes.

Esto no significó el término de las disputas con las autoridades encargadas de la persecución y sanción penal. A inicios de noviembre, y luego de que se dieran a conocer nuevos datos que daban cuenta del aumento de la delincuencia en el país, se generó una fuerte disputa entre Hinzpeter y el Ministerio Público, órgano encargado de dirigir las investigaciones de los delitos y ejercer la acción penal pública. Luego de que el jefe del MP, Sabas Chahuán, negara que la prevención del delito sea responsabilidad de su institución (sino del Ministerio del Interior), Hinzpeter reaccionó duramente, expresando una fuerte crítica al MP por su eventual falta de cumplimiento de sus obligaciones. Para ello se valió también del hecho de que en esos días el MP había desistido de pedir la prisión preventiva para tres personas que supuestamente habían participado en el homicidio de un niño a bordo de un bus en una comuna popular de Santiago. ${ }^{44}$ En

39 http://www.gob.cl/informa/2011/04/05/ministro-hinzpeter-destaco-cumplimiento-de-metas-en-materiade-seguridad-ciudadana.htm

40 http://www.emol.com/noticias/nacional/2011/10/03/506285/gobierno-emplaza-al-poder-judicial-a-tomarparte-en-discusion-por-libertades-a-encapuchados.html

41 http://www.emol.com/noticias/nacional/2011/10/03/506261/jueces-le-responden-al-presidente--es-unerror-creer-que-se-actua-discrecionalmente.html

42 http://www.cooperativa.cl/gobierno-revisara-jurisprudencia-y-criterios-de-jueces-para-decidir-ascensos / prontus_nots/2011-10-21/130029.html

43 http://www.emol.com/noticias/nacional/2011/10/22/509263/asociacion-de-jueces-acuso-presion-delgobierno-contra-el-poder-judicial.html

44 http://www.emol.com/noticias/nacional/2011/11/09/511922/hinzpeter-agudiza-polemica-con-fiscales-yjueces.html 
adelante, la disputa escaló fuertemente, ya que por una parte Hinzpeter exigió más compromiso por parte del MP en el combate de la delincuencia, además de promover la discusión de dos reformas legales, que afectarían decisivamente al MP y al Poder Judicial: el que los jueces y fiscales podrían ser electos por votación popular; y el que se traspasaran facultades de investigación de hechos delictuales desde el MP a las policías (dependientes del Ministerio del Interior). Por su parte, Chahuán insistió permanentemente en que la prevención del delito es función del Ministerio de Interior (y no del MP), a la vez que defendía el que, constitucionalmente, el MP es un órgano autónomo, y que no correspondía que el Ejecutivo pretendiera "pautear" a órganos estatales autónomos. ${ }^{45}$ Chahuán recibió en esta polémica el apoyo de la Corte Suprema, repitiendo la idea de que al gobierno no le corresponde intervenir en la acción de entes autónomos.

Al igual que en el caso de la disputa con el Poder Judicial, el gobierno y el MP optaron en definitiva por distender la disputa, aun cuando ciertamente quedó planteada una diferencia importante entre el Ejecutivo por un lado y el Poder Judicial y el MP por el otro. De hecho, a fines de año, el presidente de la Corte Suprema, Milton Juica, declaró que a partir del gobierno de Piñera había una presión política mayor sobre los jueces que en los gobiernos anteriores, y ello por el "fracaso de una promesa de seguridad que no se ha cumplido". ${ }^{46}$

\section{Evaluación general y perspectivas para el 2012}

El año 2011 estuvo dominado por las movilizaciones sociales, que tuvieron gran impacto sobre el proceso político desde distintas perspectivas. Por una parte, puso de manifiesto la debilidad del gobierno para enfrentar este tipo de situaciones, así como la incapacidad de los partidos de gobierno y de oposición para guiar el proceso de movilización social. Los partidos y el gobierno aparecen desconectados de la ciudadanía y sus intereses. En este sentido, el 2011 dio señales de la existencia de un profundo problema de representación del sistema político chileno. Las personas se sienten cada vez más lejos de los partidos y estos no muestran signos de reaccionar adecuadamente a este problema.

Por otra parte, el 2011 refleja un cambio sustantivo en las actitudes político-sociales de buena parte de los chilenos, que, a diferencia de lo que había ocurrido desde 1990, han recurrido intensa y extensamente a la protesta como mecanismo de acción política. ¿Por qué en 2011 y no antes? Es difícil saberlo, aun cuando en lo que se refiere al movimiento estudiantil, estas estrategias de movilización habían sido utilizadas anteriormente. Durante el 2011 los estudiantes mostraron una mayor capacidad de acción colectiva y pudieron mantener esa acción por un período prolongado de tiempo. Posibles hipótesis respecto de por qué esto fue así pueden estar asociadas a las características del liderazgo, a demandas que son transversales socialmente, lo que permite amplio apoyo de la ciudadanía y por la expectativa generada por el discurso gubernamental sobre la materia.

46 http://www.emol.com/noticias/nacional/2011/12/07/516140/juica-hoy-hay-una-presion-politica-mayorque-en-la-epoca-de-la-vuelta-a-la-democracia.html 
Con todo, al mismo tiempo hay que subrayar que estas movilizaciones masivas no han sido muy efectivas en términos de sus resultados y no sabemos si se mantendrán en el futuro. La pregunta hacia adelante es si este proceso de movilización está fundamentalmente acotado al problema educacional o si refleja la emergencia de nuevos temas -que habían estado sumergidos- y que podrían convertirse en nuevos ejes en torno a los cuales se configure la competencia política de Chile.

Este distanciamiento entre la política y los electores se expresa también en la caída de la aprobación del gobierno (llegando a un histórico 23\%) y de los niveles de confianza en las instituciones. Este fenómeno -como se observa en la Figura 1- es generalizado, y particularmente grave para las instituciones políticas. Esto se ve reforzado por las cifras de apoyo a la democracia que muestran una caída en la evaluación positiva sobre el funcionamiento de la democracia en Chile (desde un 26\% en 2010 a un 16\% en 2011) y un aumento de la evaluación negativa (que llega al 29\% en 2011, versus un $13 \%$ en 2010) (CEP, 2011 y Consorcio, 2010).

En suma, durante el 2011 aparecieron indicios o señales de que el sistema político chileno puede estar cambiando: por un lado, se observa una sociedad más dispuesta a organizarse y manifestarse en forma autónoma en defensa y promoción de sus intereses. De esta manera, la sociedad chilena exhibe hoy un grado de politización relativamente

Figura 1: Confianza en Instituciones (\% Mucha + Bastante)

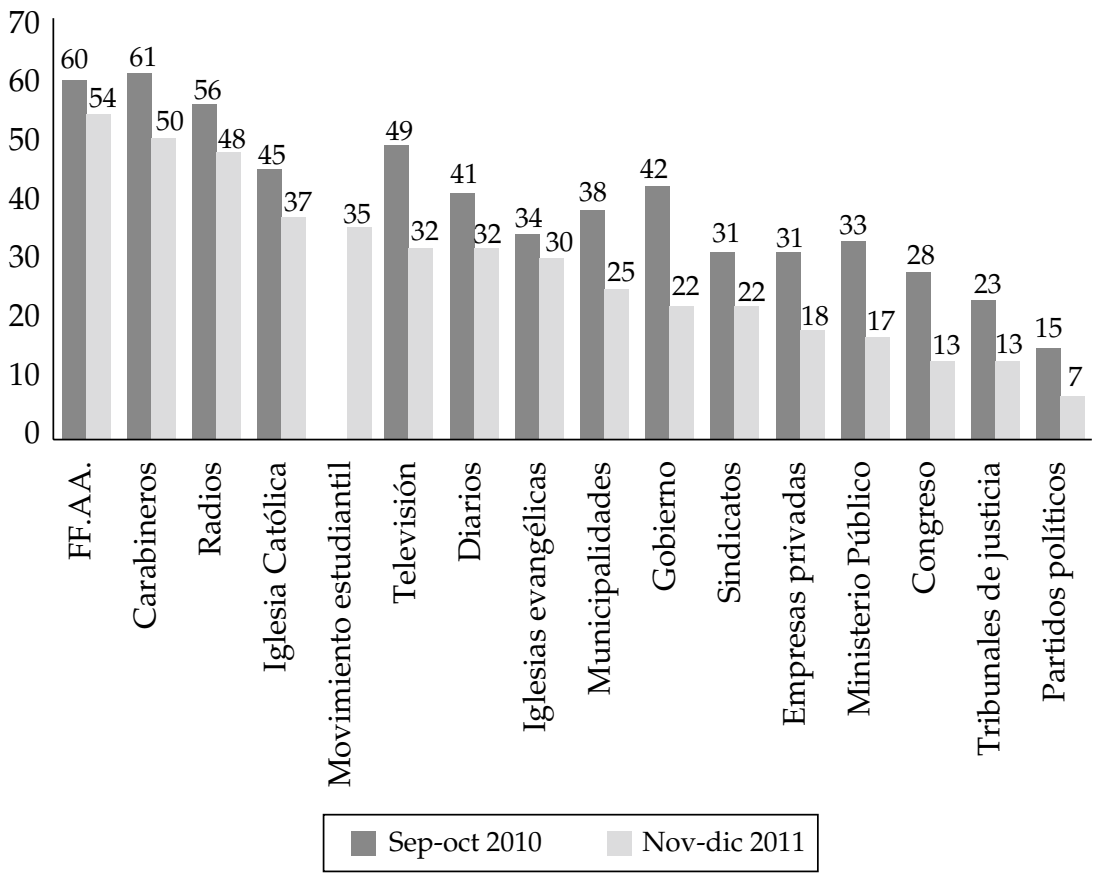

Fuente: Encuesta CEP Noviembre-Diciembre 2011 y Consorcio Septiembre-Octubre 2010. 
alto, demostrando gran interés en la resolución de problemas públicos, y demandando colectivamente soluciones a problemas que le son de primera importancia. Por el otro, se observan instituciones políticas cada vez menos capaces de entender y liderar el proceso político. Asimismo, importantes instituciones políticas están bajo fuerte crítica. A consecuencia, a futuro se configura un escenario de alta incertidumbre donde no sabemos si la sociedad seguirá en su proceso de autonomización, si los líderes e instituciones políticas lograrán hacerse cargo de estos procesos o, tal vez, que nada ocurra, manteniéndose un escenario de continuidad.

En el corto plazo, los sucesos del 2011 acrecientan la natural incertidumbre de lo que pueda ocurrir en los próximos procesos electorales de 2012 y 2013, lo que es reforzado con las reformas institucionales sobre inscripción automática y voto voluntario. En la práctica, los acontecimientos del 2011 y lo que indican sobre posibles cambios en la sociedad chilena, se constituyen como un gran desafío para los líderes políticos, los partidos y las instituciones democráticas. De ellos depende recuperar la legitimidad y el apoyo público, así como una mejor conexión con esta (aparentemente) nueva sociedad chilena, y de esta manera revitalizar la democracia.

\section{REFERENCIAS}

Cámara de Diputados. 2011. “Resumen Estadístico 2011”. Disponible en: http:/ / www.camara.cl/camara/ media/docs/estadisticas/resumen_11.pdf

Cámara de Diputados. 2010. “Resumen Estadístico 2010". Disponible en: http:/ / www.camara.cl/camara/ media/docs/estadisticas/resumen_10.pdf

Castiglioni, Rossana. 2010. "Chile y el Giro Electoral: La Vergüenza de Haber Sido y el Dolor de Ya no Ser". Revista de Ciencia Política 30 (2): 231-248.

CONFECH. 2011. "Petitorio Confech". Actualizado al 26 de junio. Disponible en: http:/ / fech.cl/ blog/2011/07/28/compilado-documentos-de-interes-movilizaciones-2011-2/petitorio-confech/

Corbo, Vittorio. 2012. “El Momento Económico Internacional y Nacional. Enero 2012". Puntos de Referencia $\mathrm{n}^{\circ}$ 341. Santiago: CEP.

Hinzpeter, Rodrigo. 2011. "Cuenta Pública del Ministerio del Interior y Seguridad Pública". Santiago, 28 de diciembre de 2011. Disponible en: http:/ / www.gob.cl/cuentas-publicas-ministeriales-2011/ ministerio-del-interior-y-seguridad-publica-cuenta-anual-2011/

INE. 2012. "Empleo Trimestral". Boletín Estadístico no 159. Santiago: Instituto Nacional de Estadísticas.

INE. 2012. "Índices de Precios al Consumidor". Boletín Estadístico n ${ }^{\circ} 158$. Santiago: Instituto Nacional de Estadísticas.

Larraín, Felipe. 2011. "Cuenta Pública del Ministerio de Hacienda". Santiago, 15 de diciembre de 2011. Disponible en: http://www.gob.cl/cuentas-publicas-ministeriales-2011/ ministerio-de-hacienda-cuenta-anual-2011/

Mardones, Rodrigo. 2007. “Chile: Todas Íbamos a ser Reinas". Revista de Ciencia Política, 27: 79-96.

Mardones, Rodrigo. 2008. "Chile: Transantiago Recargado". Revista de Ciencia Política, 28 (1): 103-119.

Moreno, Alfredo. 2011. "Cuenta Pública Anual del Ministerio de Relaciones Exteriores". Santiago, 19 de diciembre de 2011. Disponible en: http:/ / www.minrel.gob.cl/prontus_minrel/site/ artic/20111219/pags/20111219165443.php

Pérez, Cecilia. 2012. "Cuenta Pública Regional 2011. Región Metropolitana de Santiago". 12 de enero de 2012. Disponible en: http://www.gobiernosantiago.cl/CUENTA_PUBLICA_2011.pdf

Piñera, Sebastián. 2011. "Mensaje presidencial. 21 de mayo de 2011". Disponible en: http:/ / www.gob.cl/ media/2011/05/mensaje-presidencial-21-de-mayo-2011.pdf 
Carolina Segovia. Socióloga de la P. Universidad Católica de Chile y doctora en Ciencia Política de la Universidad de Michigan (Ann Arbor, EE.UU.). Actualmente es Coordinadora del Programa de Opinión Pública del Centro de Estudios Públicos (CEP) y profesora de la Escuela de Ciencia Política de la Universidad Diego Portales.

E-mail: csegovia@cepchile.cl

Ricardo Gamboa. Abogado de la P. Universidad Católica de Chile y doctor en Ciencia Política de la Universidad de Tübingen, Alemania. Actualmente es profesor asistente del Instituto de Estudios Internacionales de la Universidad de Chile.

E-mail: rgamboa@uchile.cl 
